Supplement of

\title{
Intensification characteristics of hydroclimatic extremes in the Asia monsoon region under 1.5 and $2.0{ }^{\circ} \mathrm{C}$ of global warming
}

5 Jeong-Bae Kim ${ }^{1}$, Deg-Hyo Bae ${ }^{1}$

${ }^{1}$ Department of Civil and Environmental Engineering, Sejong University, Seoul, 05006, Korea

Correspondence to: Deg-Hyo Bae(dhbae@sejong.ac.kr)

10 Contents of this file

Table S1, S2

Figures S1, S2 


\section{Table Captions}

Table S1. Results of GCM evaluation based on the skill score method.

Table S2. Statistical results of the VIC model validation at the selected basins in this study. 
Table S1. Results of GCM evaluation based on the skill score method.

\begin{tabular}{|c|c|c|c|c|c|c|c|c|c|c|c|c|c|c|c|c|}
\hline \multirow{2}{*}{ No. } & \multirow{2}{*}{ GCMs } & \multicolumn{12}{|c|}{ Score of each variable } & \multirow{2}{*}{$\begin{array}{l}\text { Total } \\
\text { score }\end{array}$} & \multirow[b]{2}{*}{ Rank } & \multirow{2}{*}{$\begin{array}{l}\text { Select } \\
(\mathrm{O}, \mathrm{X})\end{array}$} \\
\hline & & $\mathrm{pr}$ & $\begin{array}{l}\operatorname{tas} \\
\max \end{array}$ & $\begin{array}{l}\operatorname{tas} \\
\min \end{array}$ & rlut & psl & tos & ta & tas & $\mathrm{zg}$ & hus & ua & va & & & \\
\hline 1 & ACCESS1.0 & 1 & 1 & 0 & 1 & -1 & 0 & 1 & -1 & -1 & -1 & 1 & 1 & 2 & 8 & $\mathrm{X}$ \\
\hline 2 & ACCESS 1.3 & -1 & -1 & 1 & 0 & -1 & 0 & 1 & -1 & -1 & -1 & 1 & 1 & -2 & 13 & $\mathrm{X}$ \\
\hline 3 & bcc-csm1-1 & -1 & -1 & -1 & -1 & 1 & 0 & -1 & 1 & 1 & 1 & 0 & 0 & -1 & 12 & $\mathrm{X}$ \\
\hline 4 & bcc-csm1-1-m & 1 & 1 & 1 & 1 & 1 & 0 & 1 & 1 & 1 & 1 & -1 & 1 & 9 & 2 & $\mathrm{O}$ \\
\hline 5 & BNU-ESM & 1 & -1 & -1 & 1 & 1 & 1 & -1 & 1 & 1 & 1 & -1 & 0 & 3 & 7 & $\mathrm{X}$ \\
\hline 6 & CanESM2 & 1 & -1 & -1 & 1 & -1 & 1 & 1 & 1 & 1 & 1 & 1 & 1 & 6 & 5 & $\mathrm{O}$ \\
\hline 7 & CMCC-CM & -1 & 1 & 1 & 0 & -1 & 0 & 1 & 1 & 1 & 1 & 1 & 1 & 6 & 6 & $\mathrm{X}$ \\
\hline 8 & CMCC-CMS & 1 & 1 & 1 & 0 & -1 & 0 & 1 & 1 & 1 & 1 & 1 & 1 & 8 & 4 & $\mathrm{O}$ \\
\hline 9 & CNRM-CM5 & 1 & 1 & -1 & 1 & 1 & 0 & 1 & 1 & 1 & 1 & 1 & 1 & 9 & 3 & $\mathrm{O}$ \\
\hline 10 & GFDL-CM3 & 1 & 1 & 1 & 1 & -1 & 0 & 1 & -1 & -1 & -1 & 1 & 0 & 2 & 9 & $\mathrm{X}$ \\
\hline 11 & GFDL-ESM2G & 0 & 1 & -1 & 1 & -1 & 0 & -1 & -1 & -1 & -1 & 1 & 1 & -2 & 14 & $\mathrm{X}$ \\
\hline 12 & HadGEM2-CC & 1 & 1 & 1 & 1 & -1 & 1 & -1 & -1 & -1 & -1 & -1 & 1 & 0 & 11 & $\mathrm{X}$ \\
\hline 13 & IPSL-CM5A-LR & -1 & 0 & 1 & -1 & 0 & 0 & 1 & -1 & -1 & -1 & -1 & -1 & -5 & 16 & $X$ \\
\hline 14 & IPSL-CM5A-MR & -1 & 1 & 1 & -1 & 0 & 0 & 1 & -1 & -1 & -1 & 0 & -1 & -3 & 15 & $\mathrm{X}$ \\
\hline 15 & IPSL-CM5B-LR & -1 & -1 & -1 & -1 & 0 & 0 & -1 & -1 & -1 & -1 & -1 & -1 & -10 & 19 & $\mathrm{X}$ \\
\hline 16 & MIROC-ESM & -1 & -1 & -1 & 0 & 0 & 1 & -1 & -1 & -1 & -1 & -1 & -1 & -8 & 18 & $\mathrm{X}$ \\
\hline 17 & $\begin{array}{l}\text { MIROC-ESM- } \\
\text { CHEM }\end{array}$ & -1 & -1 & -1 & 1 & 0 & 1 & -1 & -1 & -1 & -1 & -1 & -1 & -7 & 17 & $\mathrm{X}$ \\
\hline 18 & MRI-CGCM3 & -1 & 0 & 1 & -1 & 1 & 1 & 1 & -1 & -1 & -1 & 1 & 1 & 1 & 10 & $X$ \\
\hline 19 & NorESM1-M & 0 & 1 & 1 & 1 & 1 & 1 & 1 & 1 & 1 & 1 & 1 & 0 & 10 & 1 & $\mathrm{O}$ \\
\hline
\end{tabular}


25 Table S2. Statistical results of the VIC model validation at the selected basins in this study.

\begin{tabular}{|c|c|c|c|c|c|c|c|}
\hline \multirow{2}{*}{ No. } & \multirow{2}{*}{ Basin } & GRDC station & \multirow{2}{*}{$\begin{array}{c}\text { Area } \\
\left(\mathrm{km}^{2}\right)\end{array}$} & \multicolumn{5}{|c|}{ Statistics } \\
\cline { 5 - 8 } & & & CC & ME & $\begin{array}{c}\text { RMSE } \\
(\mathrm{mm} / \mathrm{month})\end{array}$ & VE $(\%)$ \\
\hline 1 & Tone & Fukawa & 12,458 & 0.91 & 0.68 & 24.6 & 33.62 \\
\hline 2 & Yodo & Hirakata & 7,281 & 0.87 & 0.75 & 28.6 & -8.31 \\
\hline 3 & Gan Jiang & Jian & 56,200 & 0.93 & 0.84 & 26.8 & -12.5 \\
\hline 4 & Han Shui & Ankang & 41,400 & 0.95 & 0.84 & 17.7 & 15.8 \\
\hline 5 & Muar & Buluh Kasap & 3,130 & 0.58 & 0.33 & 62.0 & 9.2 \\
\hline 6 & Ganges & Hardinge Bridge & 846,300 & 0.86 & 0.59 & 29.3 & -32.2 \\
\hline
\end{tabular}




\section{Figure Captions}

Figure S1. Six River basins for validation for the VIC model in this study.

Figure S2. Time series of measured runoff and simulated runoff averaged at each grid in six River basins suggested in Table

S2. The simulated runoff obtained from the VIC model fed by observational meteorological input on a monthly basis (unit: $\mathrm{mm})$. The blue bars in the upper $\mathrm{x}$-axis indicate the mean areal precipitation averaged at each grid within the individual River basins. 


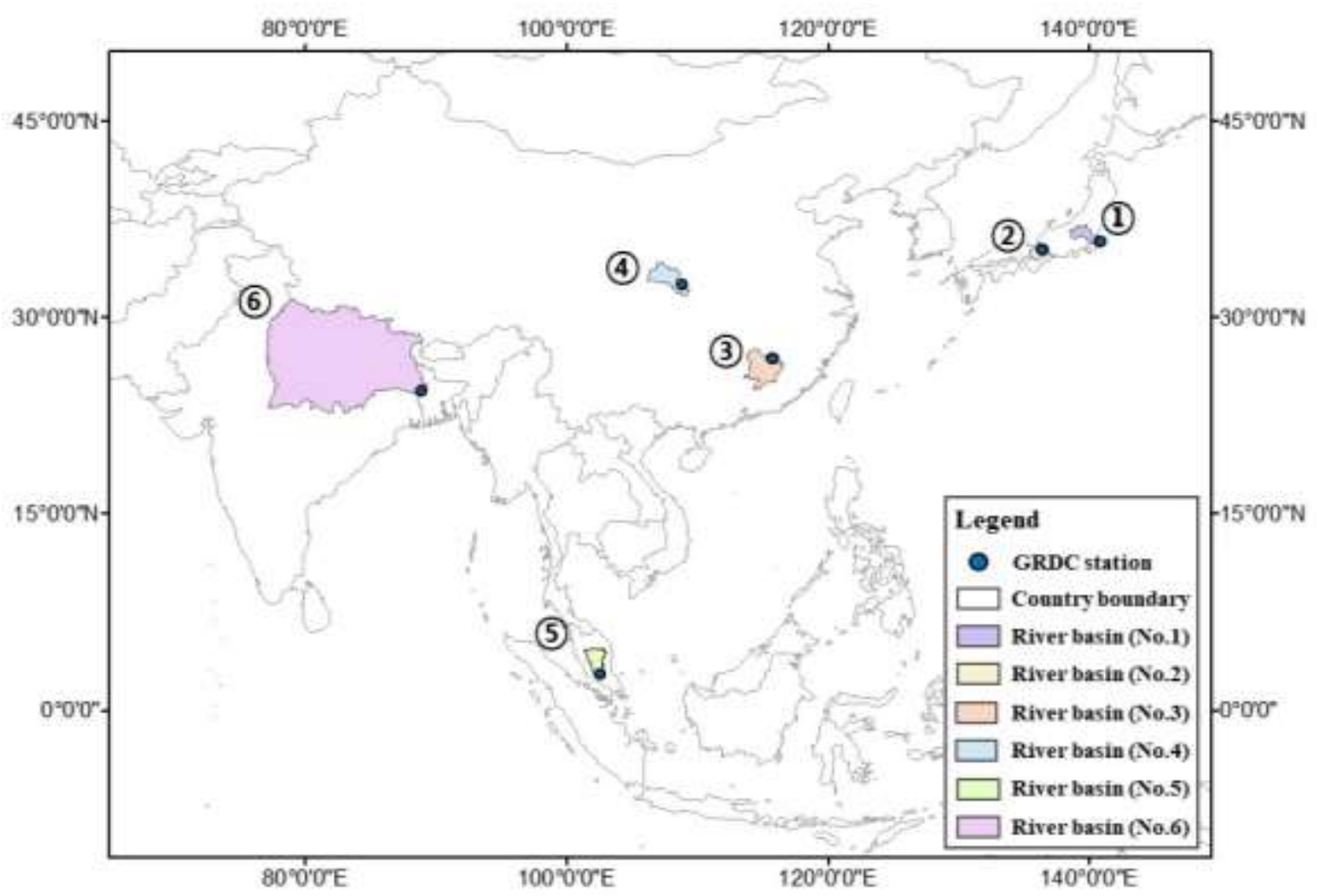

35 Figure S1: Six River basins for validation for the VIC model in this study. 
(a)

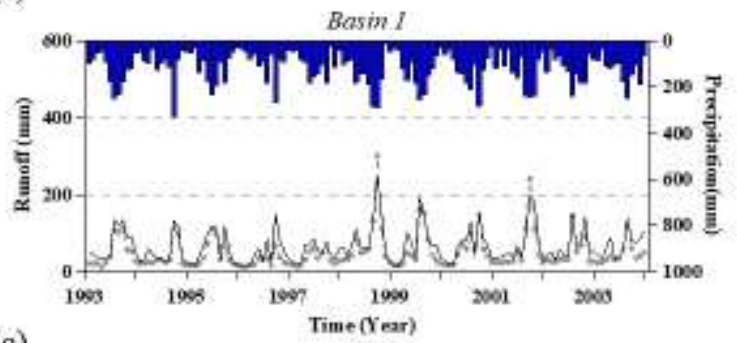

(c)

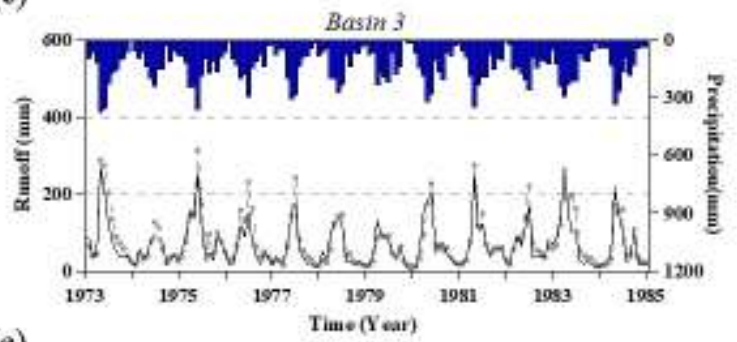

(e)

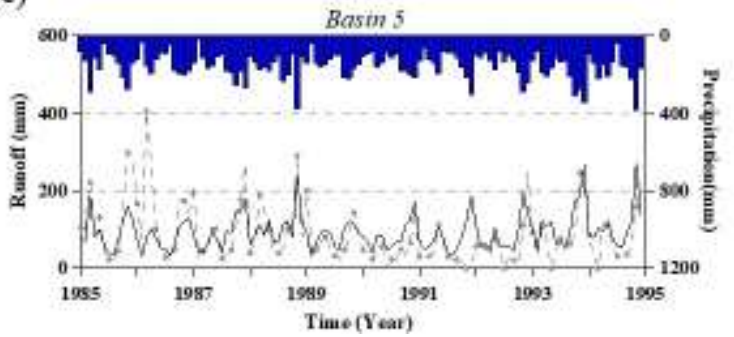

(b)

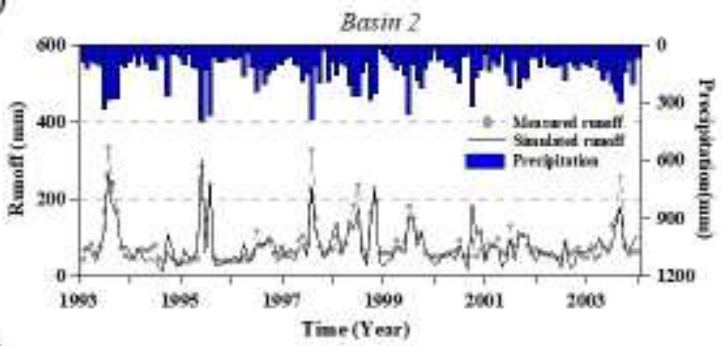

(d)

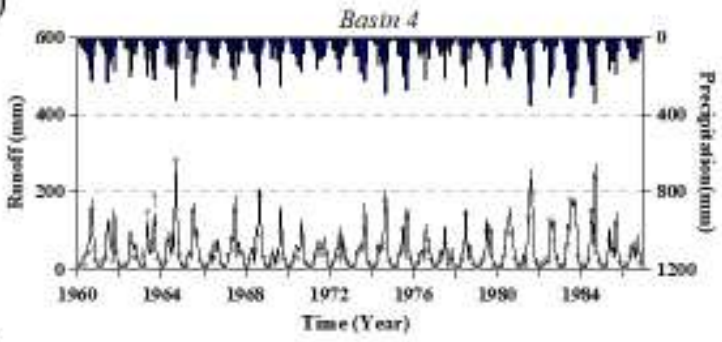

(f)

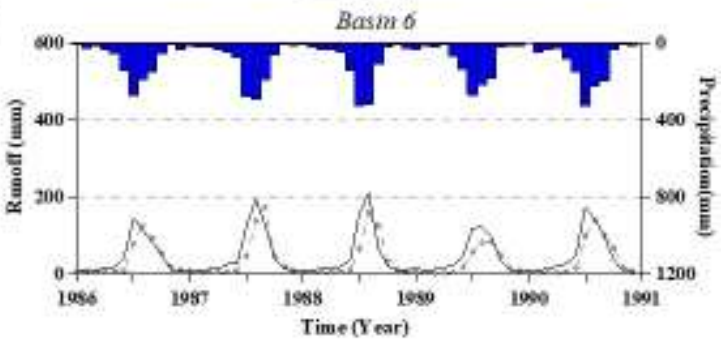

Figure S2: Time series of measured runoff and simulated runoff averaged at each grid in six River basins suggested in Table S2. The simulated runoff obtained from the VIC model fed by observational meteorological input on a monthly basis (unit: mm). The blue bars in the upper $x$-axis indicate the mean areal precipitation averaged at each grid within the individual River basins. 